\title{
Article
}

\section{Inhaltsvalidierung des Fragebogens «The Management of Aggression in People with Dementia Attitude Questionnaire German Version» (MAPDAQ-G) [Content validation of the questionnaire "German Version of the Management of Aggression in People with Dementia Attitude Questionnaire" (G-V-MAPDAQ)]}

Ryser, Agnes Elisa, Duxbury, Joy and Hahn, Sabine Available at http://clok.uclan.ac.uk/13734/

Ryser, Agnes Elisa, Duxbury, Joy ORCID: 0000-0002-1772-6874 and Hahn, Sabine ORCID: 0000-0002-2697-2014 (2015) Inhaltsvalidierung des Fragebogens «The Management of Aggression in People with Dementia Attitude Questionnaire German Version» (MAPDAQ-G) [Content validation of the questionnaire "German Version of the Management of Aggression in People with Dementia Attitude Questionnaire" (G-V-MAPDAQ)]. Pflege, 28 (3). pp. 157-168. ISSN 1012-5302

It is advisable to refer to the publisher's version if you intend to cite from the work. http://dx.doi.org/10.1024/1012-5302/a000423

For more information about UCLan's research in this area go to http://www.uclan.ac.uk/researchgroups/ and search for <name of research Group>.

For information about Research generally at UCLan please go to http://www.uclan.ac.uk/research/

All outputs in CLoK are protected by Intellectual Property Rights law, including

CLoK

Central Lancashire online Knowledge www.clok.uclan.ac.uk

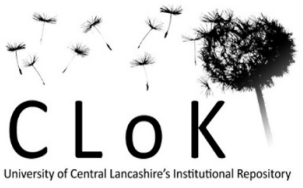




\section{CLoK}

Central Lancashire online Knowledge www.clok.uclan.ac.uk 
Inhaltsvalidierung des Fragebogens "German Version of the Management of Aggression in People with Dementia Attitude Questionnaire" (G-V-MAPDAQ)

Masterthesis

Autorin: $\quad$ Agnes Elisa Ryser

Matrikelnummer: $\quad 08-645-640$

Fachhochschule: Berner Fachhochschule Fachbereich Gesundheit

Studiengang: $\quad$ Master of Science in Pflege - Berufsbegleitender Studiengang 2010

Referentin: Prof. Dr. Sabine Hahn

Co-Referentin: Prof. Dr. Eva-Maria Panfil

Ort/ Datum: Bern, 23.01.2013

Auftraggeber: $\quad$ Abteilung Angewandte Forschung \& Entwicklung Pflege, Berner Fachhochschule Gesundheit, Bern 
Inhaltsverzeichnis

Titelblatt

$\begin{array}{ll}\text { Schnellleseüberblick } & 1\end{array}$

Zusammenfassung 2

Abstract 3

Einleitung $\quad 4$

$\begin{array}{ll}\text { Methode } & 7\end{array}$

$\begin{array}{ll}\text { Expertenbefragungen } & 7\end{array}$

$\begin{array}{ll}\text { Stichprobe } & 7\end{array}$

$\begin{array}{ll}\text { Datensammlung } & 7\end{array}$

$\begin{array}{ll}\text { Datenanalyse } & 8\end{array}$

$\begin{array}{ll}\text { Kognitive Interviews } & 8\end{array}$

$\begin{array}{ll}\text { Stichprobe } & 8\end{array}$

Datensammlung 9

Datenanalyse 9

$\begin{array}{ll}\text { Ethik } & 9\end{array}$

$\begin{array}{ll}\text { Ergebnisse } & 9\end{array}$

$\begin{array}{ll}\text { Expertenbefragungen } & 9\end{array}$

$\begin{array}{ll}\text { Kognitive Interviews } & 10\end{array}$

$\begin{array}{ll}\text { Diskussion } & 12\end{array}$

$\begin{array}{ll}\text { Limitationen } & 15\end{array}$

$\begin{array}{ll}\text { Schlussfolgerungen } & 15\end{array}$

$\begin{array}{ll}\text { Literaturverzeichnis } & 16\end{array}$

Anhang 
Berner Fachhochschule Fachbereich Gesundheit

Inhaltsvalidierung des Fragebogens "German Version of the Management of Aggression in People with Dementia Attitude Questionnaire" (G-V-MAPDAQ)

Content validation of the questionnaire "German Version of the Management of Aggression in People with Dementia Attitude Questionnaire" (G-V-MAPDAQ)

Inhaltsvalidierung des G-V-MAPDAQ

Autorin: $\quad$ Agnes Elisa Ryser, RN, BScN, cand. MScN

Auftraggeber: $\quad$ Abteilung Angewandte Forschung \& Entwicklung Pflege, Berner Fachhochschule Gesundheit, Bern

Förderer(innen): $\quad$ Schweizerische Alzheimervereinigung

Korrespondenzadresse: Agnes Elisa Ryser, cand. MScN

Gesellschaftsstrasse 90

$\mathrm{CH}-3012$ Bern

rysea1@bfh.ch

\section{Danksagung}

Ich danke Prof. Dr. Sabine Hahn und Prof. Dr. Eva-Maria Panfil für die konstruktive Begleitung. Ebenfalls bedanke ich mich herzlich bei allen Expert(inn)en und Pflegepersonen, mit deren Mitarbeit die Studie erst möglich wurde. Mein besonderer Dank gilt der Schweizerischen Alzheimervereinigung, die diese Studie grosszügig finanziell unterstützte.

AR: Rekrutierung der Teilnehmer, Datenerhebung, Transkription und Datenanalyse. 


\section{Schnellleseüberblick}

Was ist (zu dieser Thematik) schon bekannt?

In der Schweiz gibt es bisher den aus dem Englischen übersetzten "German Version of the Management of Aggression in People with Dementia Attitude Questionnaire" (G-V-MAPDAQ).

Was ist neu?

Der G-V-MAPDAQ wurde auf Inhaltsvalidität, Augenscheinvalidität und Verständlichkeit überprüft.

Welche Konsequenzen haben die Ergebnisse für die Pflegepraxis?

Der G-V-MAPDAQ ist für Pflegepersonen verständlich und zeigt eine gute Inhaltsvalidität. 


\section{Zusammenfassung}

Demenzerkrankungen zählen zu den häufigsten Erkrankungen des Alters und führen zu einer verstärkten Pflegebedürttigkeit. Ein Hauptsymptom der Demenz sind Aggressionen, welche die Haltung und den Umgang von Pflegepersonen mit Menschen mit Demenz beeinflussen. Um diese Haltung zu erfassen, entwickelten Pulsford, Duxbury und Hadi (2010) für englische Pflegeheime den "The Management of Aggression in People with Dementia Attitude Questionnaire" (MAPDAQ). In der Schweiz wurde der MAPDAQ ins Deutsche übersetzt (G-V-MAPDAQ = German Version of MAPDAQ), aber bisher noch nicht auf seine Validität und Reliabilität getestet.

Die vorliegende Studie überprüfte deshalb die Inhaltsvalidität, die Augenscheinvalidität sowie die Verständlichkeit und einheitliche Interpretierbarkeit des G-V-MAPDAQ im schweizerischen Kontext.

Die Überprüfung erfolgte durch Expertenbefragungen und mehrere kognitive Interviews mit Pflegepersonen in Pflegeheimen und psychiatrischen Kliniken.

Gemäss Experten ist der optimierte G-V-MAPDAQ gut verständlich (88\%) und zeigt eine gute Inhaltsvalidität auf (I-CVI=0,6-0,1; S-CVI-Ave=0,88). 15 Items wurden angepasst und mit 16 Pflegepersonen überprüft. Schlussendlich ist der G-VMAPDAQ für Pflegepersonen bis auf zwei Items verständlich und einheitlich interpretierbar.

Ein verständliches Instrument ermöglicht den Pflegepersonen, ihre eigene Haltung gegenüber dem Aggressionsmanagement von Menschen mit Demenz zu reflektieren und auf die Pflegequalität Einfluss zu nehmen. Der G-V-MAPDAQ könnte mit einer grösseren Stichprobe statistisch auf Validität und Reliabilität getestet werden.

Schlüsselbegriffe: Kognitive Interviews, Inhaltsvalidität, Haltung von Pflegepersonen, Aggressionsmanagement, Instrument 


\section{Abstract}

Dementia is one of the most common diseases of aging and leads to an increased need for care. A major symptom of dementia is aggression, which influences the attitude of caregivers and the interaction between them and people with dementia. To examine this attitude, Pulsford, Duxbury and Hadi (2010) developed "The Management of Aggression in People with Dementia Attitude Questionnaire" (MAPDAQ) for English nursing homes. In Switzerland, the MAPDAQ has been translated into German (G-V-MAPDAQ = German Version of MAPDAQ), but not yet tested for its validity and reliability.

Therefore the present study examined the content validity, the face validity, comprehensibility and interpretability of G-V-MAPDAQ in a Swiss context.

The review was based on expert surveys and several cognitive interviews with caregivers in nursing homes and psychiatric hospitals.

According to experts, the optimised G-V-MAPDAQ is well comprehensible (88\%) and shows good content validity $(\mathrm{I}-\mathrm{CVI}=0,6-0,1 ; \mathrm{S}-\mathrm{CVI}-\mathrm{Ave}=0,88) .15$ items have been adapted and checked with 16 nurses. Finally, apart from two items, the G-VMAPDAQ is comprehensible for nurses and can be interpreted consistently.

A comprehensible questionnaire allows nurses to reflect in practice upon their own attitude with regard to the management of aggression in people with dementia and to influence the quality of care. The G-V-MAPDAQ could be statistically tested for validity and reliability using a larger sample.

key words: cognitive interviews, content validity, management of aggression, caregivers attitude, questionnaire 


\section{Einleitung}

Demenzerkrankungen zählen zu den häufigsten Erkrankungen des Alters und beinhalten neben vermindertem Erinnerungs-, Orientierungs- und Kommunikationsvermögen weitere Symptome wie Persönlichkeitsveränderungen, Halluzinationen, Depressionen, Angst, Unruhe, Kontrollverlust und Aggressionen (BAG, 2011; Höpflinger, Bayer-Oglesby \& Zumbrunn, 2010). Verbale und physische Aggressionen im Kontext von Demenz sind für Pflegepersonen ${ }^{1}$ belastend und herausfordernd (Zeller \& Heinzer, 2010). Trotzdem ist es für Pflegepersonen unüblich, Aggressionen zu thematisieren. Zudem zeigt die Literatur, dass noch wenig über aggressives Verhalten von Menschen mit Demenz bekannt ist und für Pflegepersonen eher wenig Präventions- und Interventionsprogramme im Umgang mit aggressivem Verhalten angeboten werden (Hahn \& Metzenthin, 2010; Pulsford \& Duxbury, 2006; Skovdahl, Kihlgren \& Kihlgren, 2003; Zeller, Hahn, Needham, Kok, Dussen \& Halfens, 2009). Weil fundiertes Wissen fehlt, wenden Pflegepersonen oft die von innen mehrheitlich als sinnvoll betrachteten, bekannten freiheitsbeschränkenden Zwangsmassnahmen wie sedierende Medikation, Isolation, Ignorieren von Protesten und Fixation mit Gurten an (Becker \& Hahn, 2011; Duxbury, 2002; Franz, Zeh, Schablon, Kuhnert \& Nienhaus, 2010; Pulsford, Duxbury \& Hadi, 2010).

Vermutlich wird der Einsatz von Zwangsmassnahmen auch von der Haltung von Pflegepersonen gegenüber der Aggression bestimmt. Dieser Zusammenhang zwischen Haltung und Handlung wird von Duxbury (2002) in drei Modellen veranschaulicht. Das (1) «Internale Modell» bedeutet, dass die Haltung darin besteht, dass Aggression von Menschen mit Demenz durch Faktoren wie die Krankheit oder die Persönlichkeit ausgelöst wird. Im (2) «Externale Modell» werden in der Haltung Umgebungsfaktoren wie enge Räume, Schichtarbeit und wenig Rückzugsmöglichkeiten als Ursache für Aggression verantwortlich gemacht. Eine Haltung in der das (3) «Situative Modell» im Vordergrund steht, setzt den Schwerpunkt als Aggressionsauslöser bei der Interaktionen zwischen Menschen mit Demenz und Pflegepersonen. Gemäss Duxbury (2002) hat das letztgenannte Modell den grössten Einfluss auf das Aggressions- und Gewaltmanagement. Weiter beschreiben Pulsford et al. (2010), dass die Haltung von Pflegepersonen auch

${ }^{1}$ Pflegepersonen steht für alle in der direkten Pflege tätigen Personen mit unterschiedlichen (Zusatz-)Ausbildungen und (Berufs-)Erfahrungen. 
dadurch beeinflusst wird, ob sie eine personenzentrierte oder eine traditionelle, standardisierte Perspektive vorziehen. Bei der personenzentrierten Perspektive geht man davon aus, dass neben den neurologischen und neuropsychiatrischen Faktoren noch weitere die Demenzerkrankung beeinflussen, wie zum Beispiel die Biographie, die Persönlichkeit und auch die Interaktion mit Mitmenschen. Pflegepersonen die sich gut ausgebildet fühlen, bevorzugen eine personenzentrierte Pflege, die sich auf den ganzen Menschen und die übriggebliebenen kognitiven Fähigkeiten bezieht, anstatt sich auf deren Verluste und das Krankheitsbild zu fixieren (Epp, 2003; Zimmermann, Williams, Reed, Bonstani, Preisser \& Heck, 2005).

Aufgrund der demographischen Entwicklung mit der steigenden Zahl alter Menschen und folglich der Zunahme von Menschen mit Demenz wird das Aggressionsmanagement immer wichtiger werden (Obsan, 2011). Man vermutet, dass die zukünftige Generation älterer Menschen ausbildungsbedingt intellektuell flexibler bleibt und darum die kognitiven Einbussen länger kompensieren kann (Höpflinger et al., 2011). Dadurch verlängern sich die leichten bis mittelschweren Demenzphasen, in denen der Verlust von kognitiven Fähigkeiten bemerkt wird. Menschen mit Demenz reagieren auf den Verlust mit Stimmungsschwankungen (Höpflinger et al., 2011), was einen negativen Einfluss auf das Aggressions- und Gewaltmanagement von Pflegepersonen ausüben kann (Brodaty, Draper \& Low, 2003). Eine positive Haltung gegenüber dem Aggressionsmanagement beeinflusst einerseits die Gesundheit und das Wohlbefinden der Pflegepersonen selber, andererseits aber auch die Gesundheit, das Wohlergehen, die Pflege und die Pflegequalität der Erkrankten (Norbergh, Helin, Dahl, Hellzén \& Asplund, 2006).

Um die Reflektion von Pflegepersonen bezüglich ihrer Haltung gegenüber dem Aggressions- und Gewaltmanagement von Menschen mit Demenz zu erleichtern, ist es wichtig, diese Haltung bei Pflegepersonen zu erfassen. Zudem wird eine angepasste Pflegequalität im Demenzbereich gefördert, indem der Zusammenhang zwischen Haltung und Qualität verdeutlicht wird. Daher entwickelten Pulsford et al. (2010) in England den "The Management of Aggression in People with Dementia Attitude Questionnaire" (MAPDAQ); dieser dient der Erfassung der Haltung von Pflegepersonen zum Aggressions- und Gewaltmanagement von Menschen mit Demenz. 
Der MAPDAQ wurde aus dem vorhandenen "Management of Aggression and Violence Attitude Scale", MAVAS, (Duxbury, 2002) entwickelt und für den Gebrauch in Pflegeheimen adaptiert. Der MAPDAQ, ein Selbsteinschätzungsbogen mit 20 Items, hat einen Pearson-Testwert von $r=0,817$ und verzeichnet damit eine hohe Reliabilität (Pulsford et al., 2010). Die Items bilden die Dimensionen «Ursachen für aggressives Verhalten» und «Umgang mit aggressivem Verhalten» ab. Eine Faktoranalyse erfasst zwei Hauptfaktoren, eine personenzentrierte Perspektive (auf den Patienten/Verhalten eingehen; Eigenwert $=4,284$ ) und eine standardisierte Perspektive (Patient mit Medikation/Zwang behandeln; Eigenwert=2,639), wodurch die Konstruktvalidität des MAPDAQ gegeben ist (Pulsford et al., 2010). Im deutschsprachigen Raum existiert kein vergleichbares Instrument. Daher wurde der MAPDAQ von zwei Personen mit deutscher Muttersprache und sehr guten Englischkenntnissen ins Deutsche übersetzt und anschliessend von zwei Aggressionsexperten überprüft und fachsprachlich angepasst. Danach wurde die Übersetzung von zwei Personen mit englischer Muttersprache und guten Deutschkenntnissen ins Englische zurückübersetzt und erneut von den Experten überprüft und angepasst. Der Übersetzungsprozess folgte dem Vorgehen nach Beaton, Bombardier, Guillemin und Ferraz (2002), Koller, Aaronson, Blazeby, Bottomley, Dewolf, Fayers et al. (2007) und Wang, Lee und Fetzer (2006). Im Vergleich zu der Originalversion des MAPDAQ zeigte die Rückübersetzung nur geringe Diskrepanzen und wurde auf die in der Schweiz geläufigen Begrifflichkeiten überprüft und angepasst. Diese erste vorliegende deutschsprachige Version "German Version of the Management of Aggression in People with Dementia Attitude Questionnaire" (G-V-MAPDAQ) wurde nicht auf Validität und Reliabilität getestet.

Um den Fragebogen im deutschsprachigen Raum einsetzen zu können, ist es wichtig zu überprüfen, ob die Übersetzung sprachlich und kulturell äquivalent zu der englischen Version ist. Er muss von den Anwendern einheitlich verstanden und interpretiert werden können (Koller et al., 2007; Westat, n.d.). Mit einem schlecht übersetzten Fragebogen besteht das Risiko falsche Resultate zu generieren, während ein valides Instrument durch objektive Messungen genaue Daten und Informationen liefern kann (Wild, Grove, Martin, Eremenco, McElroy, Verjee-Lorenz et al., 2005; Polit \& Beck, 2012). 
Ziel der vorliegenden Studie ist es deshalb, den in deutscher Sprache übersetzte GV-MAPDAQ auf Inhaltsvalidität und Augenscheinvalidität (Polit \& Beck, 2012) zu überprüfen. Zudem wird der Frage nach dem einheitlichen Verständnis und der konsistenten Interpretation der Items im schweizerischen Kontext nachgegangen (Prüfer \& Rexroth, 2005).

\section{Methode}

In einem zweistufigen Verfahren wurden die Inhaltsvalidität und die Augenscheinvalidität des G-V-MAPDAQ bestimmt. In einem ersten Schritt wurden in zwei Befragungsrunden mit Experten (Polit \& Beck, 2012) die Relevanz und die Verständlichkeit des G-V-MAPDAQ ermittelt. In einem zweiten Schritt wurden kognitive Interviews geführt. Mit kognitiven Techniken wie Paraphrasieren, Hinterfragen von Antworten und Nachfragen zum Verständnis wird gezielt und systematisch überprüft, ob die Items von den Pflegepersonen eindeutig verstanden und einheitlich interpretiert werden (Prüfer \& Rexroth, 2005).

\section{Expertenbefragungen}

\section{Stichprobe}

Rekrutiert wurden die Experten über eine Schlüsselperson mit vertiefen Kenntnissen im Feld der Untersuchung sowie über soziale Netzwerke. Einschlusskriterien waren: 10 bis 15 Experten mit Pflegediplom, Erfahrungswissen und Fachwissen zu Demenz und Aggression, Arbeitsplatz in psychiatrischen Kliniken, Pflegeheimen oder Organisationen, die sich mit Demenz oder Aggression befassen.

\section{Datensammlung}

Via E-Mail erhielten die Experten ein Informationsschreiben zum Vorgehen und einen halbstrukturierten Fragebogen (Abbildung 1). Mit einem zweistufigen Antwortformat (Ja/Nein) beurteilten die Experten die Verständlichkeit der Items des G-V-MAPDAQ (Klaus, 2007). Die Relevanz beurteilten sie anhand einer vierstufigen Likertskala (1=gar nicht, 2=wenig, 3=ziemlich, 4=sehr). Zusätzlich bestand für die Experten die Möglichkeit, Verbesserungsvorschläge zu den Items abzugeben, so dass diese nach jeder Runde optimiert werden konnten. Demographische Daten der Experten für die Stichprobenbeschreibung wurden erhoben. Die Expertenbefragungen erfolgten von Mitte September bis Mitte November 2012. 


\section{Datenanalyse}

Die Relevanz der Items wurde mit dem Content Validity Index (CVI) berechnet, der unterteilt wird in den Item Content Validity Index (I-CVI; Relevanz für das einzelne Item) und den Scale Content Validity Index/Average Method (S-CVI/Ave; Relevanz für den gesamten MAPDAQ (Polit \& Beck, 2012). Die vierstufige Likertskala wurde dichotomisiert zu nicht relevant ( $1=$ gar nicht, $2=$ =wenig) und relevant ( $3=z i e m l i c h$, 4=sehr). Die Anzahl der von den Experten als relevant beurteilten Items wurde dann durch die gesamte Anzahl Experten geteilt. Items mit einem I-CVI von $<0,8$ wurden gemäss den Expertenempfehlungen angepasst (Polit \& Beck, 2012).

Der S-CVI/Ave für den G-V-MAPDAQ wurde mit dem Durchschnitt der I-CVI Werte berechnet. Ein S-CVI/Ave von $>0,90$ zeigt eine ausgezeichnete Inhaltsvalidität (Polit \& Beck, 2012).

Die Verständlichkeit der Items wurde im zweistufigen Antwortformat (Ja/Nein) erfasst, deskriptiv dargestellt und deshalb in relativen Häufigkeiten berechnet (Müller, 2011). Items mit einer Verständlichkeit $<80 \%$ oder Verbesserungsvorschlägen wurden trotz I-CVI Werten $>0,8$ nach der ersten Befragungsrunde angepasst, da eine mangelnde Verständlichkeit die Zuverlässigkeit des G-V-MAPDAQ negativ beeinflusst.

\section{Kognitive Interviews}

\section{Stichprobe}

Angefragt wurden 5 Pflegeheime und 5 psychiatrische Kliniken. Die Rekrutierung erfolgte über die Pflegedienstleitungen. Einschlusskriterien waren: 10 bis 15 deutschsprachige Pflegepersonen (Prüfer \& Rexroth, 2005) mit verschiedenen (Zusatz-)Ausbildungen und (Berufs-)Erfahrungen (Otto, 2010), die Menschen mit Demenz betreuen und Erfahrung mit verbaler und/oder physischer Patienten- bzw. Bewohneraggression haben. 


\section{Datensammlung}

Die kognitiven Interviews wurden mit dem aus den Expertenbefragungen optimierten G-V-MAPDAQ und einem semistrukturierten Evaluationsfragebogen durchgeführt. Der Evaluationsfragebogen enthielt alle Items des G-V-MAPDAQ und die kognitiven Techniken. Die Einzelinterviews wurden während jeweils 30 bis 40 Minuten in den Institutionen vor Ort geführt. Zuerst füllten die Pflegepersonen den optimierten G-VMAPDAQ aus, um die Verständlichkeit der Items in der Anwendung zu überprüfen. Dann wurde die Verständlichkeit und Interpretierbarkeit der einzelnen Items mit dem Evaluationsfragebogen anhand der kognitiven Techniken nachgefragt. Die Interviews fanden zwischen November und Dezember 2012 statt.

\section{Datenanalyse}

Die Interviews wurden in Anlehnung an Willis (1999) selektiv transkribiert. Die Transkriptionen wurden den jeweiligen Items zugeordnet, paraphrasiert und mit Zitaten belegt. Problematische Items wurden identifiziert und anhand der Zitate für die jeweils nächsten Interviewrunden angepasst. Die Arbeitsschritte wurden im Forschungsteam besprochen und reflektiert, um die Glaubwürdigkeit der Datenanalyse zu erhöhen (Flick, Kardoff \& Steinke, 2008).

\section{Ethik}

Die Ethikkomission des Kantons Bern wurde angefragt und erklärte sich nicht zuständig für die Studie, weil es sich bei den Beteiligten um Berufskolleg(inn)en handelte. Die Beteiligten wurden schriftlich und mündlich aufgeklärt, ihr Einverständnis lag schriftlich vor und die Studienteilnahme war freiwillig. Der Datenschutz wurde durch Anonymität der Daten gewährleistet. Den Beteiligten entstanden keine negativen Konsequenzen und sie konnten die Teilnahme jederzeit grundlos abbrechen.

\section{Ergebnisse}

\section{Expertenbefragungen}

In der ersten Befragungsrunde beteiligten sich 11, in der zweiten 10 Experten mit beruflichen Qualifikationen von Höherer Fachausbildung Stufe $1^{2}$ bis zu Habilitation in Soziologie. Die Experten verfügten über 6 bis 37 Jahre Berufserfahrung und im

\footnotetext{
${ }^{2}$ Vertiefende Fachausbildung für diplomiertes Pflegepersonal, mit verschiedenen Schwerpunkten, beispielsweise gerontologische Pflege (SBK, 2013)
} 
Schnitt über 12 Jahre Erfahrung im Demenzbereich sowie 20 Jahre Erfahrung im Aggressionsbereich. 7 von 12 Experten verfügten über eine Weiterbildung in einem der beiden Bereiche (Tabelle 2).

Tabelle 2 im Anhang

In der ersten Befragungsrunde stuften die Experten 14 Items als nicht sehr relevant ein (I-CVI=0,54-0,72). Diese Items wurden anschliessend gemäss den

Expertenempfehlungen optimiert und aus Item 7 wurden zwei Items (7a; 7b) gebildet (Tabelle 3). In Runde zwei stuften die Experten noch drei Items (5, 7b, 19) als nicht sehr relevant ein ( $I-C V I=0,6-0,71)$; diese wurden gemäss der

Expertenrückmeldungen erneut optimiert. Der resultierende S-CVI/Ave erreichte danach zwar nicht den von Polit \& Beck (2012) geforderten Wert von 0,90, war aber mit 0,88 ein zufriedenstellender Wert (Davis, 1992).

Die durchschnittliche Verständlichkeit der Items betrug nach Einschätzung der Experten in Runde eins 72\% und erreichte in Runde zwei, nach den Verbesserungen, 88\%. Damit war die Verständlichkeit des gesamten G-V-MAPDAQ genügend gut, um mit den kognitiven Interviews beginnen zu können.

Tabelle 3 im Anhang

Kognitive Interviews

Die Pflegepersonen (2 Männer, 14 Frauen) mit einer Ausbildung zu Pflegeassistenz bis zu Höherer Fachausbildung Stufe 1, waren im Schnitt 42 Jahre alt und bildeten sich mehrheitlich $(n=11 ; 10)$ in den Bereichen Demenz und/oder Aggression weiter (Tabelle 4). Die Mehrheit ( $n=15)$ erlebte im letzten Monat Aggressionen von Menschen mit Demenz. 4 von 16 Pflegepersonen haben einen Migrationshintergrund.

Tabelle 4 im Anhang 
In drei Befragungsrunden mit jeweils 11, 3 und 2 Pflegepersonen, wurde ein aus den Ergebnissen der vorhergehenden Interviewrunden angepasster Evaluationsfragebogen und optimierter G-V-MAPDAQ eingesetzt.

Neun Items $(3,4,6,7 b, 8,12,14,15,17)$ wurden in Runde eins als unverständlich in Begrifflichkeiten und Satzstellungen beurteilt, besonders von Pflegepersonen mit Migrationshintergrund; die Items mussten daher angepasst werden. Tabelle 5 zeigt den Verlauf der Item-Anpassungen über die Runden hinweg auf.

Tabelle 5 im Anhang

Drei Items (4, 6 und 15) blieben über mehrere Runden unverständlich und werden näher erläutert.

Item 4 Menschen mit Demenz können aufgrund verschiedener krankheitsbedingter Faktoren aggressiv werden, interpretieren die Befragten als somatische Krankheit: "Zum Beispiel Schmerzen haben irgendwo, eine Blasenentzündung (...) zu der Demenz hinzu (...) oder Zahnweh" (5/20:59). Obwohl der Begriff krankheitsbedingte Faktoren von den Experten als verständlich eingestuft wurde, zeigte er sich bei der Überprüfung mit Pflegepersonen ungeeignet und wurde zu Menschen mit Demenz können aufgrund ihrer Krankheit aggressiv werden angepasst. In Runde zwei wird Krankheit zum Teil noch als somatisches Leiden gedeutet und deshalb der Begriff Krankheit durch Demenz ersetzt.

Item 6 Das Personal sollte die Möglichkeit haben, aggressive Heimbewohner in separaten Räumen zu isolieren zeigte, dass Isolation unterschiedlich interpretiert wird: "Also wie ein Isolationszimmer. Wir haben hier auch die IVZ" (1/16:50); "Kann ein Einzelzimmer sein, sein eigenes Zimmer (...) eine ruhigere Ecke (...) eine Nische wo es ruhiger ist (...)" (6/12:47). Das Item wurde angepasst zu Das Personal sollte die Möglichkeit haben, aggressive Heimbewohner in Räumen zu isolieren. Der Begriff Isolation wurde weiter verschieden aufgefasst: "(...) oder in der Stube versuchen wir es manchmal auch (...) das Patientenzimmer kann es auch noch sein (...)" (13/13:41). Das Item wurde für Runde drei angepasst zu: Das Personal sollte die Möglichkeit haben, aggressive Heimbewohner zu isolieren. Dennoch wurde Isolation weiterhin als jemanden aus dem Geschehen herausnehmen verstanden: "(...) also Isolieren heisst für mich nicht, sie ins Zimmer zu sperren (...)" (16/16:12). 
Item 15 Auf Aggression könnte in meiner Institution wirkungsvoller reagiert werden verwirt mit den Begriffen Institution und wirkungsvoller: "(...) wie wirkungsvoller reagieren? Mit was für Medikamenten oder mit Validieren oder mit Alternativen" (3/34:21). Das Item wurde angepasst zu Auf Aggression könnte in meiner Abteilung wirkungsvoller reagiert werden. In Runde zwei wird wirkungsvoller meist als deeskalierendes Vorgehen aufgefasst, ist aber dennoch unklar: "Dass man deeskalierend Arbeiten kann"; "Wirkungsvoller (...) das ist nicht so gut (...)" (14/39:40). Wirkungsvoller wurde darum wie in der deutschen Originalversion durch effektiver ersetzt und in Runde drei verstanden als: "(..) das versteh ich unter effektiv, dass wir so geschult sind, dass uns das nicht aus dem Konzept bringt" (16/29:47) oder dass man besser reagieren könnte.

\section{Diskussion}

Nach aktuellem Wissen ist dies die erste Studie, die sich mit der Inhaltsvalidität, der Augenscheinvalidität sowie der Frage nach dem einheitliche Verständnis und der konsistenten Interpretation des G-V-MAPDAQ im schweizerischen Kontext befasst. Die Ergebnisse zeigen, dass die Originalversion des G-V-MAPDAQ einige unklare Items mit viel Interpretationsspielraum aufwies.

Auffallend ist, dass in den Expertenbefragungen gewisse Items trotz eines hohen ICVI eine niedrige Verständlichkeit zeigten und umgekehrt. Beispielsweise ist Item 7 in Runde eins relevant (I-CVI=0,9) aber mit $45 \%$ nicht verständlich. Deshalb wurde es aufgrund der Expertenempfehlungen in zwei Items, 7a und 7b, unterteilt (Tabelle 3). Es ist jedoch zu beachten, dass die Relevanz des Items 7 erst durch die Zweiteilung von 0,9 (7) auf 0,8 (7a) und 0,6 (7b) sank, wodurch sich allerdings die Verständlichkeit von 45\% (7) auf 100\% (7a) und 80\% (7b) erhöhte. In Runde zwei wurde besonders Item $7 \mathrm{~b}$ als nicht relevant $(\mathrm{I}-\mathrm{CVI}=0,6)$ beurteilt, verzeichnete aber mit $80 \%$ eine gute Verständlichkeit. Weil das Ziel der Studie in erster Linie der Verständlichkeit und einheitlichen Interpretation der Items galt, ist ein S-CVI/Ave von 0,88 gut (Davis, 1992), obwohl Polit und Beck (2012) einen Wert von 0,9 vorziehen. Item 7 erfragt, ob Personen zur eigenen Sicherheit und zur Sicherheit anderer mit Zwangsmassnahmen behandelt werden sollen. Möglicherweise wird Item $7 \mathrm{~b}$ von den Experten als nicht relevant eingestuft, weil es nach dem Einsatz von Zwangsmassnahmen zur Sicherheit anderer fragt. Aggressionen gegen 
Pflegepersonen werden aber vielfach als Teil der Arbeit angesehen, die nicht zu Zwangsmassnahmen legitimieren (Pulsford \& Duxbury, 2006). Pflegepersonen stellten erstaunlicherweise die Relevanz des Items nicht in Frage, wurden aber auch nicht explizit danach gefragt.

Nach den Expertenbefragungen wurden unklare Items anhand des Inhaltsvaliditätsindex, der Verständlichkeit und den Expertenempfehlungen optimiert und nach den Anpassungen von den Experten als gut befunden. In der ersten Runde der kognitiven Interviews, in der vier von 16 Pflegepersonen einen Migrationshintergrund aufwiesen, zeigte diese Gruppe jedoch Verständnisschwierigkeiten in Bezug auf einzelne Begriffe. Das könnte darauf zurückzuführen sein, dass ihre Muttersprache nicht Deutsch ist. Nach Peters und Passchier (2005) soll aber das Instrument einfach zu verstehen und anzuwenden sein und deshalb der Sprache und Kultur angepasst werden. Dies bestätigen Westat (n.d.), Beaton et al. (2002) und Koller et al. (2007), die auf mögliche sprachliche und kulturelle Verständnisschwierigkeiten hinweisen. Wang et al. (2010) ergänzen, dass ein Austauschen von wortwörtlichen Begriffen nicht automatisch zu Äquivalenz und einer besseren Verständlichkeit der Items führt. Gemäss Obsan (2010) bilden Pflegepersonen mit Migrationshintergrund heute und zukünftig im Altersbereich eine grosse Gruppe. Daher ist es wichtig, dass der G-V-MAPDAQ von dieser Gruppe angewendet werden kann, weshalb die unverständlichen Items angepasst wurden.

Weiter fiel auf, dass der Begriff krankheitsbedingte Faktoren von den Pflegepersonen mehrheitlich als somatische Erkrankung wie Schenkelhalsfraktur, Harnwegsinfekt oder Zahnschmerzen verstanden wird. Vermutlich wird dieses Verständnis einerseits dadurch beeinflusst, dass Pflegepersonen ein personenzentriertes Paradigma vorziehen (Pulsford \& Duxbury, 2006; Pulsford et al., 2010) und neben den neurologischen und neuropsychiatrischen Aspekten der Demenz weitere Faktoren als Ursache für Aggressionen halten (Stokes, 2000). Andererseits zeigen beispielsweise Maier und Mayer (2012) oder Kitwood (2008), dass Menschen mit Demenz aufgrund ihrer Krankheit ihre Schmerzen verbal schlecht äussern können und deshalb oft mit Aggressionen reagieren. Aufgrund der Ergebnisse sollte der G-VMAPDAQ diesen Aspekt möglicherweise in einem weiteren Item zusätzlich erfassen.

Unklar bleibt Item 6 (Tabelle 5). Isolation wird von den Pflegepersonen sehr unterschiedlich verstanden, von Einschliessen von Menschen mit Demenz in ein 
Isolationszimmer bis hin zu Herausnahme aus dem Geschehen. Laut Pflegepersonen dürfen in einzelnen Institutionen Zwangsmassnahmen nur beschränkt angewendet werden. Ebenfalls sind spezielle Räumlichkeiten wie Isolationszimmer in gewissen Institutionen nicht vorhanden. Diese Aussagen verdeutlichen die bisher in der Schweiz existierende uneinheitliche Regelung und Sichtweise im Umgang mit freiheitsbeschränkenden Massnahmen (BAG, 2011). Die Einführung des neuen Erwachsenenschutzrechtes könnte möglicherweise etwas daran ändern, indem beispielsweise neu alle Institutionen verpflichtet sind, bewegungseinschränkende Massnahmen zu protokollieren (Curaviva, 2012). Item 6 scheint jedoch alle oben genannten Sichtweisen zu erfassen. Deshalb wäre es sinnvoll, dass Pflegepersonen bei Item 6 zusätzlich beschreiben könnten, was sie unter Isolation verstehen.

Interessant ist in diesem Zusammenhang weiter, dass für Pflegepersonen Items, die eine standardisierte Perspektive abbilden, mehrheitlich schwieriger zu verstehen waren und öfters angepasst werden mussten, als Items, die eine personenzentrierte Perspektive abfragten. Das könnte darauf zurückzuführen sein, dass Pflegepersonen die Ursachen für aggressives Verhalten externen, internen oder situationsabhängigen Faktoren zuschreiben (Duxbury, 2002; Pulsford \& Duxbury, 2006). Andererseits kann auch der Umgang mit aggressivem Verhalten innerhalb der Institution das Verständnis der Items erschweren, weil die eigene Haltung und die standardisierten Vorgehen im Widerspruch zueinander stehen (Duxbury \& Whittington, 2004). Hapell und Harrow (2010) unterstreichen, dass der Einsatz von Isolationsmassnahmen mit der Arbeitsplatzkultur zusammenhängt. Im Hinblick auf die Umsetzung des neuen Erwachsenenschutzrechts erscheint deshalb ein Instrument, das die Haltung von Pflegepersonen erfassen kann, umso wichtiger.

Item 15 (Tabelle 5) zeigt ebenfalls, dass die unterschiedliche Praxis, die Arbeitsplatzkultur und die Haltung von Pflegpersonen das Verständnis des Items beeinflussen. Dieses Item wurde in der ersten Befragungsrunde völlig unterschiedlich verstanden, von der Verabreichung höherer Dosen an Medikamenten bis hin zu deeskalierenden Massnahmen. Pulsford \& Duxbury (2006) beschreiben, dass Pflegepersonen in Pflegeheimen und psychiatrischen Kliniken dazu neigen, anderen Mitteln als Zwangsmassnahmen den Vorzug zu geben. Hingegen zeigen Jansen, Middel, Dassen und Reijneveld (2009), dass in Spitälern medizinische Massnahmen 
bevorzugt werden. Dies verdeutlicht, dass die Haltung gegenüber dem Gewalt- und Aggressionsmanagement von Menschen mit Demenz institutionsabhängig sein kann. Der G-V-MAPDAQ müsste deshalb idealerweise auch erfassen können, was für ein Management in der jeweiligen Institution üblich ist.

\section{Limitationen}

Die Stichprobengrösse in den Interviewrunden zwei und drei war klein und aufgrund mangelnder Zeitressourcen fand kein Membercheck ${ }^{3}$ statt (Flick et al., 2008). Jedoch wurde die Interviewsituation analysiert und im Sinne der Authentizität mit den Äusserungen der Befragten sorgfältig umgegangen (Flick et al., 2008). Daher sollte die Übertragbarkeit der Ergebnisse trotz der kleinen Stichprobe gewährleistet sein.

\section{Schlussfolgerung}

Die Studie zeigt, dass der optimierte G-V-MAPDAQ bis auf die Items 6 und 15 für Pflegepersonen in Pflegeheimen und psychiatrischen Kliniken verständlich und einheitlich interpretierbar ist. Pflegepersonen können anhand des G-V-MAPDAQ zum Thema Haltung sensibilisiert werden und ihre eigene Haltung gegenüber dem Aggressions- und Gewaltmanagement von Menschen mit Demenz reflektieren. Noch ist der G-V-MAPDAQ in der Praxis eingeschränkt anwendbar. Deshalb sollte Item 6 um die Möglichkeit ergänzt werden anzugeben, was Pflegepersonen unter Isolation verstehen. Item 15 sollte zusätzlich erfassen, welches Aggressionsmanagement in den jeweiligen Institutionen eingesetzt wird. Weiter könnte ein Item angefügt werden, welches das Schmerzmanagement erfasst, beispielsweise Menschen mit Demenz werden wegen körperlicher Schmerzen aggressiv. Alle diese Items könnten mit einer grösseren Anzahl kognitiver Interviews mit Pflegepersonen evaluiert werden. Um den G-V-MAPDAQ definitiv in der Praxis einzusetzen, könnte er in einer Pilotstudie mit einer grösseren Stichprobe statistisch auf Reliabilität und Validität getestet werden. Mit einem validierten Instrument könnten standardisiert Daten erhoben und Erkenntnisse zu der Haltung für Schulungen im Bereich Lehre und Weiterbildung gewonnen werden. Die Pflegepersonen könnten veranlasst werden, mit dem Thema Haltung bewusster umzugehen und es könnten entsprechend präventive Massnahmen getroffen werden. Dadurch würde die Pflegequalität von Menschen mit Demenz positiv beeinflusst werden.

\footnotetext{
${ }^{3}$ Überprüfung der Interviews mit den Beteiligten auf Vollständigkeit, Richtigkeit und Glaubwürdigkeit der Daten
} 


\section{Literaturverzeichnis}

Bundesamt für Gesundheit (2011). Demenz.

http://www.bag.admin.ch/themen/medizin/00683/12261/index.html [19.01.2013]

Bundesamt für Gesundheit (2011). Neues Erwachsenenschutzrecht tritt am 1. Januar 2013 in Kraft.

http://www.eipd.admin.ch/content/ejpd/de/home/dokumentation/mi/2011/201101-12.html [19.01.2013]

Beaton, D.; Bombardier, C.; Guillemin, F.; \& Ferraz, M. B. (2002). Recommendations for the Cross-Cultural Adaptation of Health Status Measures. Unpublished manuscript.

Becker, S.; Hahn, S. (2011). Auch Nichtstun schränkt ein. Freiheitsbeschränkung und Zwang: Bewegungsfreiheit oder Stillstand? NOVAcura, (1), 2-4.

Brodaty, H.; Draper, B.; Low, L.F. (2003). Nursing home staff attitudes towards residents with dementia: strain and satisfaction with work. Journal of Advanced Nursing, 44 (6), 583-590.

Curaviva. (2012). Neues Erwachsenenschutzrecht. [18.01.2013]

Davis, L.L. (1992). Instrument review: Getting the most from your panel of experts. Applied Nursing Research, 5, 194-197.

Duxbury, J.; Whittington, R. (2004). Causes and management of patient aggression and violence: staff and patient perspectives. Journal of Advanced Nursing, 50 (5), 469-478.

Duxbury, J. (2002). An evaluation of staff and patient views of and strategies employed to manage inpatient aggression and violence on one mental health unit: a pluralistic design. Journal of Psychiatric and Mental Health Nursing, 9, 325-337.

Epp, T. D. (2003). Person-centered Dementia Care: A Vision to be Refinded. The Canadian Alzheimer Disease Review. 
Flick, U.; Kardoff, E.; Steinke, I. (2008). Qualitative Forschung - ein Handbuch. (6. Aufl.). Reinbek: Rowohlt .

Franz, S.; Zeh, A.; Schablon, A.; Kuhnert, S.; Nienhaus, A. (2010). Aggression and violence against health care workers in Germany - a cross sectional retrospective survey. Bio Med Central Health Services Research, 10, 51.

Hahn, S.; Metzenthin, P. (2010). Aggressive Ereignisse als Grenzsituation. NOVAcura, 20-22.

Happell, B.; Harrow, A. (2010). Nurses' attitudes to the use of seclusion: A review of the literature. International Journal of Mental Health Nursing, 19, 162-168.

Höpflinger, F.; Bayer-Oglesby, L.; Zumbrunn, A. (2001). Pflegebedürftigkeit und Langzeitpflege im Alter. Aktualisierte Szenarien für die Schweiz. Bern: Verlag Hans Huber.

Jansen, G.J.; Middel, B.; Dassen, W.N.; Reijneveld, M.S.A. (2006). Cross-Cultural Differences in Psychiatric Nurse's Attitudes to Inpatient Aggression. Archieves of Psychiatric Nursing, 20 (2), 82-93.

Kitwood, T. (2008). Demenz. Der person-zentrierte Ansatz im Umgang mit verwirrten Menschen. (5.Aufl.) Bern: Verlag Hans Huber.

Klaus, K. (2007). Mündliche und schriftliche Befragung - Ein Lehrbuch. Landau: Verlag Empirische Pädagogik.

Koller, M.; Aaronson, N. K.; Blazeby, J.; Bottomley, A.; Dewolf, L.; Fayers, P.; Johnson, C.; Ramage, J.; Scott, N.; West, K. (2007). Translation procedures for standardised quality of life questionnaires: The European Organisation for Research and Treatment of Cancer (EORTC) approach. European Journal of Cancer, 43 (12), 1810-1820.

Maier, R.; Mayer, P. (2012). Der vergessene Schmerz. München: Reinhardt Verlag.

Müller, M. (2011). Statistik für die Pflege. Handbuch für Pflegeforschung und wissenschaft. Bern: Verlag Hans Huber. 
Norbergh, K.G.; Helin, Y.; Dahl, A.; Hellzén, O.; Asplund, K. (2006). Nurses' attitudes towards people with dementia: the semantic differential technique. Nursing Ethics, 13 (3).

Otto, S. (2010). Bericht zum Personal Skill- und Grademix, mit Bezug zur Petition der Personalverbände für flankierende Massnahmen bei der DRG-Einführung. http://www.google.ch/url?sa=t\&rct=i\&q=\&esrc=s\&source=web\&cd=1\&ved=0C C8QFjAA\&url=http\%3A\%2F\%2Fwww.sbkbe.ch\%2F\%3Fredirect\%3Dgetfile.php\%26cmd\%255Bgetfile\%255D\%255Buid \%255D\%3D1145\&ei=Crz6Ulb5EMrMswbXt4HABg\&usg=AFQjCNF LwkSkG7 a3Euu7G95X LWu8PhOA\&bvm=bv.41248874,d.Yms [30.06.2012]

Peters, M.; Passchier, J. (2006). Translating Instruments for Cross-Cultural Studies in Headache Research. American Headache Society, 82-91.

Polit, D.; Beck, C. (2012). Nursing Research. Generating and Assessing Evidence for Nursing Practice. Wolters Kluwer: Lippincott.

Prüfer, P.; Rexroth, M. (2000). Zwei-Phasen-Pretesting. ZUMA-Arbeitsbericht, 221.

Prüfer, P.; Rexroth, M. (2005). Kognitive Interviews. ZUMA How-to-Reihe, 15, 321.

Pulsford, D.; Duxbury, J. (2006). Aggressive behaviour by people with dementia in residential care settings: a review. Journal of Psychiatric and Mental Health Nursing, 13, 611-618.

Pulsford, D.; Duxbury, J.; Hadi, M. (2010). A survey of staff attitudes and responses to people with dementia who are aggressive in residental care settings. Journal of Psychiatric and Mental Health Nursing, 18, 97-104.

SBK (2013). Höhere Fachausbildung in Gesundheits- und Krankenpflege, Stufe 1 (HöFa1). http://www.sbk-asi.ch/ [19.01.2013]

Schweizerisches Gesundheitsobservatorium (2010). Gesundheitspersonal in der Schweiz-Bestandesaufnahme und Perspektiven bis 2020. http://www.obsan.admin.ch/bfs/obsan/de/index/05/publikationsdatenbank.html ?publication ID=3207 [12.12.2012] 
Schweizerisches Gesundheitsobservatorium (2010). Ausländisches

Gesundheitspersonal in der Schweiz. Bericht 39.

http://www.google.ch/url?sa=t\&rct=j\&q=\&esrc=s\&source=web\&cd=2\&sqi=2\&v ed=0CDwQFjAB\&url=http\%3A\%2F\%2Fwww.obsan.admin.ch\%2Fbfs\%2Fobsa n\%2Fde\%2Findex\%2F01\%2F02.Document.137578.pdf\&ei=4JjyUPiBGtDIsga FvYDwDA\&usg=AFQjCNFCTWrjZ-qzErJsQI9WzaoQo4t1w\&sig2=OQqf5Slj5tX2Nq1ImwbPXg\&bvm=bv.1357700187,d.Yms [01.01.2013]

Stokes, G. (2000). Challenging Behaviour in Dementia: A Person Centered Approach. Winslow Editions:

Skvodahl, K.; Kihlgren, A.L.; Kihlgren, M. (2003). Different attitudes when handling aggressive behaviour in dementia-narratives from two caregiver groups. Aging \& Mental Health, 7 (4), 277-286.

Wang, W. L.; Lee, H. L.; Fetzer, S. J. (2006). Challenge and strategies of instrument translation. Western Journal Nursing Research, 28 (3), 310-321.

Westat (n.d.). Translation Guidelines for the AHRQ Surveys on Patient Safety Culture, 1-11.

Wild, D.; Grove, A.; Martin, M.; Eremenco, S.; McElroy, S.; Verjee-Lorenz, A.; Erikson, P. (2005). Principles of Good Practice for the Translation and Cultural Adaption Process for Patient-Reported Outcomes (PRO) Measures: Report of the ISPOR Task Force for Translation and Cultural Adaption. Value in Health Journal (8), 2, 94-104.

Willis, G.B. (1999). Cognitive Interviewing - A "How To" Guide. Research Triangle Institute, 1-37.

Zeller, A.; Hahn, S.; Needham, I.; Kok, G.; Dassen, T.; Halfens, R.J.G. (2009). Aggressive Behaviour of Nursing Home Residents Toward Caregivers: A Systematic Literature Review. Geriatric Nursing, 30, 174-187.

Zeller, H.; Heinzer, M. (2010). Aggression im Alters- und Pflegeheim. Ein Konzept bietet Unterstützung. NOVAcura, 9, 23-25. 
Zimmermann, S.; Williams, C.S.; Reed, P.S.; Boustani, M.; Preisser, J.S.; Heck, E.; Sloane, P.D. (2005). Attitudes, Stress, and Satisfaction of Staff Who Care for Residents With Dementia. The Gerontologist, 45, 96-105. 


\section{Anhang}

Abbildung 1. Fragen zur Beurteilung der Verständlichkeit und Relevanz eines Items des G-V-MAPDAQ (in Anlehnung an Klaus, 2007; Polit \& Beck, 2012).

\begin{tabular}{|c|}
\hline Diese Aussage ist klar und verständlich formuliert $\quad \square$ ja $\quad \square$ nein \\
\hline Für wie relevant erachten Sie diese Aussage? $\square$ gar nicht $\square$ ein wenig $\square$ ziemlich $\square$ sehr \\
\hline Kommentar: \\
\hline
\end{tabular}

Tabelle 2. Demographische Angaben Experten (Befragungsrunden 1 und 2).

\begin{tabular}{|c|c|c|c|c|}
\hline Charakteristika & Range & Mittelwert & Anzahl & (Häufigkeiten \%) \\
\hline Teilnehmer gesamthaft & & & 12 & \\
\hline Berufserfahrung (in Jahren) & $6-37$ & 22 & & \\
\hline $\begin{array}{l}\text { Erfahrung mit Menschen mit Demenz } \\
\text { (in Jahren) }\end{array}$ & $0,5-30$ & 12 & & \\
\hline Erfahrung mit Aggression (in Jahren) & $6-37$ & 20 & & \\
\hline Geschlecht männlich & & & 8 & $(66 \%)$ \\
\hline Geschlecht weiblich & & & 4 & $(33 \%)$ \\
\hline \multicolumn{5}{|l|}{ Bildungsabschluss } \\
\hline Höhere Fachausbildung Stufe 1 (Höfa1) & & & 2 & $(16 \%)$ \\
\hline Höhere Fachausbildung Stufe 2 (Höfa 2) & & & 1 & $(8 \%)$ \\
\hline Bachelor in Pflegewissenschaften & & & 1 & $(8 \%)$ \\
\hline Master in Pflegewissenschaften & & & 2 & $(16 \%)$ \\
\hline Doktorat in Pflegewissenschaften & & & 2 & $(16 \%)$ \\
\hline andere Bildungsabschlüsse & & & 4 & $(33 \%)$ \\
\hline Zusatzausbildung Aggression & & & 8 & $(66 \%)$ \\
\hline Dauer in Tagen & $3,5-45$ & 18 & & \\
\hline Zusatzausbildung Demenz & & & 8 & $(66 \%)$ \\
\hline Dauer in Tagen & $1-30$ & 17,5 & & \\
\hline
\end{tabular}


Tabelle 3. Expertenbefragungen: Berechnung des CVI (I-CVI und S-CVI/Ave) (Polit \& Beck, 2012).

\begin{tabular}{|c|c|c|c|c|}
\hline Fragen Runde 1 & CVI R1 & Fragen Runde 2 & CVI R2 & CVI all \\
\hline 1 & 0.72 & 1 & 0.9 & 0.9 \\
\hline 2 & 0.9 & 2 & 1 & 1 \\
\hline 3 & 0.72 & 3 & 0.88 & 0.88 \\
\hline 4 & 0.81 & 4 & 1 & 1 \\
\hline 5 & 0.54 & 5 & 0.71 & 0.71 \\
\hline 6 & 0.81 & 6 & 0.8 & 0.8 \\
\hline \multirow[t]{2}{*}{7} & \multirow[t]{2}{*}{0.9} & $7 a$ & 0.8 & 0.8 \\
\hline & & $7 b$ & 0.6 & 0.6 \\
\hline 8 & 0.9 & 8 & 1 & 1 \\
\hline 9 & 0.81 & 9 & & 0.81 \\
\hline 10 & 0.9 & 10 & 1 & 1 \\
\hline 11 & 0.9 & 11 & & 0.9 \\
\hline 12 & 0.72 & 12 & 0.9 & 0.9 \\
\hline 13 & 0.81 & 13 & 0.9 & 0.9 \\
\hline 14 & 0.9 & 14 & 1 & 1 \\
\hline 15 & 0.54 & 15 & 1 & 1 \\
\hline 16 & 0.9 & 16 & & 0.9 \\
\hline 17 & 0.9 & 17 & 0.9 & 0.9 \\
\hline 18 & 0.9 & 18 & 0.9 & 0.9 \\
\hline 19 & 0.63 & 19 & 0.7 & 0.7 \\
\hline 20 & 0.81 & 20 & & 0.81 \\
\hline S-CVI/ Ave & 0.8 & & 0.88 & \\
\hline S-CVI/ Ave (Befrac & & & & 0.88 \\
\hline
\end{tabular}


Tabelle 4. Demographische Angaben Pflegepersonen (Befragungsrunden 1, 2 und 3).

\begin{tabular}{|c|c|c|c|c|}
\hline Charakteristika & Range & Mittelwert & Anzahl & (Häufigkeiten \%) \\
\hline Teilnehmer & & & 16 & \\
\hline Migrationshintergrund & & & 4 & \\
\hline Berufserfahrung (in Jahren) & $0,42-35$ & 15 & & \\
\hline $\begin{array}{l}\text { Erfahrung mit Menschen mit Demenz } \\
\text { (in Jahren) }\end{array}$ & $0,17-24$ & 13 & & \\
\hline $\begin{array}{l}\text { Letztes aggressives Verhalten } \\
\text { (verbal/ physisch) erlebt von }\end{array}$ & $6-37$ & 20 & & \\
\hline \multicolumn{5}{|l|}{ Menschen mit Demenz } \\
\hline letzter Monat & & & 15 & $(94 \%)$ \\
\hline im letzten halben Jahr & & & 5 & $(31 \%)$ \\
\hline \multicolumn{5}{|l|}{ im letzten Jahr } \\
\hline \multicolumn{5}{|l|}{ liegt länger als ein Jahr zurück } \\
\hline Geschlecht männlich & & & 2 & $(12 \%)$ \\
\hline Geschlecht weiblich & & & 14 & $(88 \%)$ \\
\hline Alter in Jahren & & 42 & & \\
\hline
\end{tabular}

\begin{tabular}{|c|c|c|c|c|}
\hline \multicolumn{5}{|l|}{ Bildungsabschluss } \\
\hline Pflegeassistentin & & & 2 & $(13 \%)$ \\
\hline Pflegefachperson DNI/ FaGe & & & 2 & $(13 \%)$ \\
\hline Pflegefachperson DNII/ HF & & & 9 & $(56,25 \%)$ \\
\hline Pflegefachperson FH & & & 1 & $(6,25 \%)$ \\
\hline andere Bildungsabschlüsse & & & 2 & $(11,5 \%)$ \\
\hline Zusatzausbildung Aggression & & & 10 & $(62,5 \%)$ \\
\hline Dauer in Tagen & $1-5$ & 3,6 & & \\
\hline Zusatzausbildung Demenz & & & 11 & $(68,75 \%)$ \\
\hline Dauer in Tagen & $1-50$ & 10 & & \\
\hline
\end{tabular}


Tabelle 5. Verlauf der Item - Anpassungen.

\begin{tabular}{|c|c|c|c|}
\hline Item & Runde 1 & Runde 2 & Runde 3 \\
\hline Nr. 3 & $\begin{array}{l}\text { Menschen mit Demenz können } \\
\text { aufgrund ihrer } \\
\text { Persönlichkeitsstruktur } \\
\text { aggressiv werden }\end{array}$ & $\begin{array}{l}\text { Menschen mit Demenz können } \\
\text { aufgrund ihrer Persönlichkeit } \\
\text { aggressiv werden }\end{array}$ & Item belassen \\
\hline Nr. 4 & $\begin{array}{l}\text { Menschen mit Demenz können } \\
\text { aufgrund verschiedener } \\
\text { krankheitsbedingter Faktoren } \\
\text { aggressiv werden }\end{array}$ & $\begin{array}{l}\text { Menschen mit Demenz können } \\
\text { aufgrund ihrer Krankheit } \\
\text { aggressiv werden }\end{array}$ & $\begin{array}{l}\text { Menschen mit Demenz } \\
\text { werden aufgrund ihrer } \\
\text { Demenz aggressiv }\end{array}$ \\
\hline Nr. 6 & $\begin{array}{l}\text { Das Personal sollte die } \\
\text { Möglichkeit haben, aggressive } \\
\text { Heimbewohner in separaten } \\
\text { Räumen zu isolieren }\end{array}$ & $\begin{array}{l}\text { Das Personal sollte die } \\
\text { Möglichkeit haben, aggressive } \\
\text { Heimbewohner in Räumen zu } \\
\text { isolieren }\end{array}$ & $\begin{array}{l}\text { Das Personal sollte die } \\
\text { Möglichkeit haben, } \\
\text { aggressive Heimbewohner zu } \\
\text { isolieren }\end{array}$ \\
\hline Nr. 7b & $\begin{array}{l}\text { Aggressive Menschen mit } \\
\text { Demenz sollten zur Sicherheit } \\
\text { Anderer durch körperliche und/ } \\
\text { oder medikamentöse } \\
\text { Zwangsmassnahmen } \\
\text { eingeschränkt werden können }\end{array}$ & $\begin{array}{l}\text { Aggressive Menschen mit } \\
\text { Demenz sollten zur Sicherheit } \\
\underline{\text { anderer Personen durch }} \\
\underline{\text { körperliche und/ oder }} \\
\underline{\text { medikamentöse }} \\
\text { Zwangsmassnahmen } \\
\underline{\text { eingeschränkt werden können }}\end{array}$ & Item belassen \\
\hline Nr. 8 & $\begin{array}{l}\text { Medikamente sind eine wertvolle } \\
\text { Massnahme im Umgang mit } \\
\text { Aggression bei Menschen mit } \\
\text { Demenz }\end{array}$ & $\begin{array}{l}\text { Medikamente sind eine wichtige } \\
\text { Massnahme im Umgang mit } \\
\text { Aggression bei Menschen mit } \\
\underline{\text { Demenz }}\end{array}$ & Item belassen \\
\hline Nr. 12 & $\begin{array}{l}\text { Körperliche } \\
\text { Zwangsmassnahmen werden in } \\
\text { der Institution in der ich arbeite } \\
\text { mehr als notwendig eingesetzt }\end{array}$ & $\begin{array}{l}\text { Körperliche } \\
\text { Zwangsmassnahmen werden in } \\
\underline{\text { meiner Abteilung mehr als }} \\
\underline{\text { notwendig eingesetzt }}\end{array}$ & Item belassen \\
\hline Nr.14 & $\begin{array}{l}\text { Eine wertschätzende } \\
\text { Beziehungsgestaltung zwischen } \\
\text { dem Personal und Menschen } \\
\text { mit Demenz kann Aggression } \\
\text { verringern }\end{array}$ & $\begin{array}{l}\text { Eine wertschätzende Beziehung } \\
\text { zwischen dem Personal und } \\
\text { Menschen mit Demenz kann } \\
\text { Aggression verringern }\end{array}$ & Item belassen \\
\hline Nr. 15 & $\begin{array}{l}\text { Auf Aggression könnte in meiner } \\
\text { Institution wirkungsvoller } \\
\text { reagiert werden }\end{array}$ & $\begin{array}{l}\text { Auf Aggression könnte in meiner } \\
\text { Abteilung wirkungsvoller reagiert } \\
\text { werden }\end{array}$ & $\begin{array}{l}\text { Auf Aggression könnte in } \\
\text { meiner Abteilung effektiver } \\
\text { reagiert werden }\end{array}$ \\
\hline Nr. 17 & $\begin{array}{l}\text { Sedierende, Fix- und } \\
\text { Reservemedikation müsste } \\
\text { häufiger verwendet werden, um } \\
\text { aggressives Verhalten von } \\
\text { Menschen mit Demenz zu } \\
\text { verringern }\end{array}$ & $\begin{array}{l}\underline{\text { Sedierende Fix- und }} \\
\underline{\text { Reservemedikation müsste }} \\
\underline{\text { häufiger verwendet werden, um }} \\
\underline{\text { aggressives Verhalten von }} \\
\underline{\text { Menschen mit Demenz zu }} \\
\underline{\text { verringern. }}\end{array}$ & Item belassen \\
\hline
\end{tabular}




\section{Eidesstatliche Erklärung zur Masterthesis}

Ich,

\begin{tabular}{|l|l|l|l|}
\hline Vorname & Name & Matrikelnummer & Gesetzlicher Wohnort \\
\hline Agnes Elisa & Ryser & $08-645-640$ & Bern, BE \\
\hline
\end{tabular}

versichere hiermit an Eides statt, dass ich die vorliegende Arbeit

Inhaltsvalidierung des Fragebogens "German Version of the Management of Aggression in People with Dementia Attitude Questionnaire" (G-V-MAPDAQ)

selbständig verfasst, bisher weder ganz noch in Teilen, weder an der Berner Fachhochschule noch an einer anderen Bildungsinstitution als Prüfungsleistung vorgelegt und keine anderen als die angegebenen Hilfsmittel benutzt habe. Sämtliche Stellen der Arbeit, die benutzen Werke oder Quellen aus dem Internet im Wortlaut oder dem Sinn nach entnommen sind, habe ich durch Quellenangaben kenntlich gemacht. Dies gilt auch für sämtliche Abbildungen.

Ich bin mir bewusst, dass es sich bei Plagiarismus um schweres akademisches Fehlverhalten handelt, das Sanktionen bis zum nachträglichen Entzug des erworbenen akademischen Titels nach sich zieht.

\begin{tabular}{|l|l|l|}
\hline Ort & Datum & Eigenhändige Unterschrift \\
\hline Bern & 23. Januar 2013 & A. Ry \\
& & \\
\hline
\end{tabular}




\section{Autor(inn)enrichtlinien}

(Stand August 2012)

1. Ziele und Themenbereiche

2. Manuskripteinreichung

2.1 Peer-Review-Verfahren

2.2 Manuskriptgestaltung

2.3 Punkt-für-Punkt-Bearbeitungsprotokoll

2.4 Agreement Autor(inn)en

\section{Ziele und Themenbereiche}

«Pflege» veröffentlicht Beiträge über Aspekte der Gesundheits- und Krankenpflege sowie der Geburtshilfe und trägt zur Entwicklung der Pflegepraxis und der Pflegewissenschaft bei. Sie versteht sich als umfassendes Diskussionsforum für Themen der klinischen Pflegeforschung und der Entwicklung und Prüfung von Pflegetheorien und -konzepten in der Praxis der Pflege. «Pflege»

liefert neue Erkenntnisse aus Studien der Pflegepraxis

zeigt, wie Forschungsergebnisse in die Praxis einfließen

berichtet über Erfahrungen und Probleme im Forschungsprozess

stellt Forschungsmethoden, Anleitungen zum Lesen von Forschungsarbeiten u.a. vor diskutiert und kritisiert Konzepte, Modelle und Theorien der Pflege und beschreibt, wie sie in Forschung und Praxis implementiert werden bietet Analysen und Diskussionen aus der Pflegepraxis setzt sich auseinander mit spezifischen Aspekten der Pflege und der Entwicklung der Pflegeberufe

«Pflege» veröffentlicht Originalarbeiten, Grundsatzartikel, Forschungsberichte und Fallstudien, die neue wissenschaftliche Ergebnisse beinhalten und eine solide theoretische Basis haben. Es werden nur Arbeiten veröffentlicht, die nicht bereits anderen Zeitschriften zur Veröffentlichung angeboten oder bereits publiziert worden sind. Manuskripte, die zur Eigen- oder Firmenwerbung eingereicht werden, gelangen nicht zur Veröffentlichung. «Pflege» veröffentlicht ebenfalls Zusammenfassungen von Dissertationen, Buchbesprechungen und Mitteilungen zu Veranstaltungen und anderen interessanten Geschehnissen hinsichtlich der Thematiken der Pflege. Ein Jahrgang umfasst in der Regel sechs Hefte. «Pflege» wird vom Cumulative Index to Nursing \& Allied Health Literature (CINAHL) erfasst. Der Impact-Factor beträgt 0.182 (2011).

\section{Manuskripteinreichung}

Die Manuskripte sind online auf www.editorialmanager.com/pflege einzureichen. Das System unterstützt eine breite Palette an gängigen File-Formaten für Manuskripte: Word, WordPerfect, RTF, TXT und LaTex; für Abbildungen: TIFF, GIF, JPEG, EPS, PPT und Postscript. Open-Office-Formate werden noch nicht unterstützt. Bitte senden Sie keine Papierabzüge des Manuskripts ein. Bitte achten Sie darauf, Ihr Manuskript bei Ersteinreichung mit einem kurzen begleitenden Brief an die Herausgeberinnen zu versehen (im System in das Textfeld "Kommentare" einzugeben). In diesem formlosen Anschreiben mit der Bitte um Begutachtung versichern Sie, dass Sie das in Frage kommende Instrument der Berichterstattung bei der Anfertigung Ihres Manuskriptes berücksichtigt haben (siehe http://www.equator-network.org/resourcecentre/library-of-health-researchreporting/ (04.02.2012). 
Die eingereichten Manuskripte werden mit Ausnahme der Vorstellungen von Dissertationen, Editorials, Leserbriefe, Mitteilungen und Buchbesprechungen von Fachexpertinnen und experten in einem doppelblinden Peer-Review-Verfahren beurteilt.

\subsection{Peer-Review-Verfahren}

Die Entscheidung, ob ein eingereichtes Manuskript in den Reviewprozess geht, erfolgt auf Basis der in den Autor(inn)enrichtlinien angeführten Anforderungen durch die Herausgeberinnen. Der Reviewprozess erfolgt ausschließlich im Onlineverfahren. Sollte sich die Eignung bestätigen, wird das eingereichte Manuskript an zwei unabhängige, von den Herausgeberinnen ausgewählte Fachexperten (Referees) weitergeleitet. Die Referees haben eine beratende Funktion und unterstützen die Entscheidung der Herausgeberinnen. Gegebenenfalls wird das Manuskript zusätzlich einer statistischen Beraterin/einem statistischen Berater vorgelegt. Wenn von einem Autor oder einer Autorin für die Begutachtung ihres Manuskripts begründete Bedenken gegenüber einer/m bestimmten Referee bestehen, können diese bei der Einreichung des Manuskripts im Anschreiben an die Redaktion angeführt werden. Wenn eine Herausgeberin Co-Autorin eines eingereichten Manuskripts ist, wird sie weder in der Frage der prinzipiellen Annahme noch in der Zuteilung von Referees und der Beurteilung des Manuskripts einbezogen. Der Reviewprozess der «Pflege» erfolgt zweiseitig verblindet, $d$. h. den Referees und Autor(inn)en wird gegenseitige Anonymität und Vertraulichkeit garantiert. Um diese Anonymität gewährleisten zu können, ist während des gesamten Prozesses (von der Einreichung bis zur Schlussentscheidung über das Manuskript) auf Angaben zu Personen, Institutionen oder Projekten, die Hinweise auf die Autorenschaft enthalten, zu verzichten. Die Herausgeberinnen, die Referees und alle am Reviewprozess beteiligten Personen (Redaktionssekretariat, statistische Konsultation) wahren ebenfalls diese Prinzipien, indem sie keine Informationen über Autor(inn)en und Referees weitergeben oder Manuskripte mit Dritten diskutieren. Die Beurteilung der Manuskripte erfolgt nach den Kriterien der Originalität, der methodischen Angemessenheit, der Nachvollziehbarkeit, der kritischen Reflektion und der Fundierung und Vernetzung mit dem Stand des Wissens. Zur Erstellung sowie zur Begutachtung von Manuskripten über empirische Studien wird empfohlen, den Kriterien standardisierter Instrumente der Berichterstattung zu folgen, z. B. PRISMA, CONSORT (siehe http://www.equatornetwork.org/resource-centre/library-of-health-research-reporting/ (15.02.2010) oder http://www.sign.ac.uk/methodology/checklists.html (15.02.2010). Die Herausgeberinnen wie auch die Referees sind verpflichtet, einen Verdacht auf Plagiat zu prüfen beziehungsweise diesen der Redaktion zu melden. Ein Manuskript wird abgelehnt, wenn ein teilweises oder vollständiges Plagiat vorliegt, d. h. wenn ein Manuskript mehr oder weniger umfassende Anteile eigener Arbeiten (Selbstplagiat) oder von Arbeiten anderer Autor(inn)en ohne Angabe der Referenz beinhaltet. In einem solchen Fall behält sich die Redaktion vor, die Autor(inn)en für weitere Manuskripte zu sperren. Die zeitliche Frist richtet sich nach der Schwere des Plagiats und kann von drei Jahren bis unbefristet reichen.

\section{Ablehnung und Überarbeitung von Manuskripten}

Die zuständige Herausgeberin entscheidet unter Zuhilfenahme der Gutachten der Referees bzw. der statistischen Beratung über Ablehnung und Annahme bzw. vorläufige Annahme vorbehaltlich einer Überarbeitung des Manuskripts. Die Autor(inn)en erhalten in jedem Fall die Gutachten der Referees, es sei denn, das Manuskript ist nicht in das Peer-ReviewVerfahren eingegangen. Bei Ablehnung wird im Weiteren keine Korrespondenz geführt. Bei vorläufiger Annahme erhalten die Autor(inn)en Informationen zur weiteren Vorgangsweise, zum Beispiel zu notwendigen Überarbeitungen. Den Autor(inn)en steht es frei, die Überarbeitung vorzunehmen und das Manuskript wieder einzureichen oder das Manuskript zurückzuziehen.

\subsection{Manuskriptgestaltung}

\section{Die Einhaltung der Regeln zur Manuskriptgestaltung wird im Vorfeld durch das}

Redaktionssekretariat geprüft. Die Einhaltung der Autor(inn)enrichtlinien sowie der aktuellen Regeln zu Grammatik und Rechtschreibung wird nach endgültiger Annahme des Manuskripts durch das Editorische Controlling überprüft. 
Der Text ist satzreif online über das Manuskriptverwaltungssystem Editorial Manager ${ }^{\oplus}$ unter der Adresse www.editorialmanager.com/pflege einzureichen.

Satzreif bedeutet, dass der Text linksbündig, ohne automatische Silbentrennung, ohne Unterstreichungen und ohne automatische Aufzählungen zu verfassen ist. Für Hervorhebungen im Text die kursive Schrift bitte sparsam verwenden. Das Manuskript folgt den neuen Rechtschreibregeln und den Autor(inn)enrichtlinien. Abkürzungen sind zugunsten des interdisziplinären Verständnisses, Fußnoten zugunsten der Lesbarkeit zu vermeiden. Bitte verzichten Sie auf Kopf- und Fußzeilen.

Im Sinne von Gender Mainstreaming ist auf eine geschlechtergerechte Formulierung zu achten.

\section{Manuskripte für Originalarbeiten, Grundsatzartikel, Forschungsberichte und Fallstudien umfassen:}

Titelblatt (als gesondertes Dokument, um die Anonymität des Manuskripts zu gewährleisten; diese Informationen dürfen nicht Bestandteil des Manuskripts sein) Schnellleseüberblick (insgesamt 350-400 Zeichen)

Zusammenfassung in Deutsch (1500-1600 Zeichen inkl. Leerzeichen)

Abstract (Zusammenfassung in Englisch) (1500-1600 Zeichen inkl. Leerzeichen)

Schlüsselbegriffe (key words) in Deutsch und Englisch (3-5 Wörter)

Haupttext

Literaturverzeichnis

Abbildungen und/oder Tabellen

Das Titelblatt enthält:

Name des Instituts, an dem der Autor oder die Autorin derzeit beschäftigt ist Titel auf Deutsch und auf Englisch

Kurztitel für die Umschlagseite (max. 60 Zeichen inkl. Leerzeichen)

Namen sämtlicher Autor(inn)en mit vorangestellten Vornamen (und berufliche Qualifikation)

Namen der Auftraggeber(innen)/Förderer(innen) (Drittmittelzuwendung), wenn nicht vorhanden: explizit angeben, dass kein Auftraggeber/Förderer vorhanden war falls abweichend von dem Institut, dem der Autor/die Autorin gegenwärtig angehört: Name der Einrichtung, an dem das Projekt durchgeführt wurde falls zutreffend: konkrete Benennung des Gesamtprojektes, in dem das Manuskript angefertigt wurde Korrespondenzadresse mit akademischem Grad und Länderschlüssel Ggf. Danksagungen am Ende des Titelblatts wird der Beitrag der einzelnen Autor(inn)en (Initialen) zur vorgestellten Arbeit beschrieben (z. B. AB Datenerhebung, CD Datenanalyse, EF Projektleitung).

-Dem Text ist ein Schnellleseüberblick zu folgenden Fragen voranzustellen:

. Was ist (zu dieser Thematik) schon bekannt?

Was ist neu?

Welche Konsequenzen haben die Ergebnisse für die Pflegepraxis?

(Alle Antworten zusammen dürfen nicht mehr als 400 Zeichen inklusive Leerzeichen beanspruchen.)

- Der Titel der Arbeit und die Zusammenfassung sind für die internationale Indexierung ins Englische zu übersetzen (Abstract). Es ist dabei das britische Englisch zu verwenden (außer bei amerikanischen Namen und Bezeichnungen): z. B. programme vs. program, centre vs. center, criticise vs. criticize, colour vs. color, catalogue vs. catalog.

- Ebenso ist eine strukturierte Zusammenfassung (Fließtext) von maximal 1500-1600 Zeichen inklusive Leerzeichen zu verfassen (Struktur: Hintergrund, Fragestellung/Ziel, Methode, Ergebnisse, Schlussfolgerungen, jedoch ohne Gliederungsüberschriften)

- Schlüsselbegriffe: Der deutschen Zusammenfassung und dem englischen Abstract sind 3 bis 5 Schlüsselbegriffe (key words), die in prägnanter Weise die Schwerpunkte des 
Manuskriptinhalts beschreiben, anzufügen. Englische key words sind der Schlagwortliste in Medline http://www.nlm.nih.gov/mesh/MBrowser.html zu entnehmen.

- Umfang und Gliederung des Manuskripts: Ein Manuskript sollte zirka 8 bis 15

Manuskriptseiten zu 30 Zeilen (21 600 bis 27000 Zeichen inkl. Leerzeichen) in 12-PunktSchrift umfassen und mit 1,5-zeiligem Abstand geschrieben werden.

Die Gliederung der Arbeit soll den internationalen Gepflogenheiten entsprechen:

Einleitung, Frage-/Problemstellung, Ziel, Methode und Material, Ergebnisse, Diskussion.

Der Text soll durch Untertitel ohne Nummerierungen gegliedert sein. Überschriften kurz

(drei bis sechs Worte) und einheitlich halten. Maximal zwei Gliederungsstufen (Dekaden) verwenden.

Im gesamten Text ist auf Hinweise zur Autorenschaft zu verzichten. Das ganze

Manuskript ist - mit Ausnahme des Deckblattes - als eine gesamte Datei ins System zu stellen; lediglich Tabellen und Abbildungen, die ein anderes Format aufweisen, sind als separate Dateien einzugeben.

- Literaturangaben im Fließtext werden für Einzelautor(inn)en (Meyer, 2010), Zwei-AutorenWerke (Müller \& Stein, 2009) und Mehrautorenwerke (Stein, Müller \& Meyer, 2008; gilt für bis zu sechs Autor(inn)en bei Erstnennung der Quelle) wie in den Beispielen erstellt. Werke mit mehr als sechs Autor(inn)en werden bei Erstnennung bis zum sechsten Verfasser aufgeführt und dann mit «et al.» abgekürzt (Schmitt, Müller, Stein, Holz, Berger, Friedrich et al., 2007). Bei erneuter Nennung werden alle Mehrautorenwerke (ab drei Autor(inn)en) nach dem erstgenannten Autor(inn)en mit «et al.» abgekürzt (Stein et al. 2008; Schmitt et al., 2007).

- Tabellen und Abbildungen werden fortlaufend nummeriert; sie sind mit kurzen Legenden zu versehen. Zur Ausrichtung der Tabellenspalten keine Leerzeichen verwenden. Im Manuskript ist ihr Standort anzugeben.

- Literaturverzeichnis: Es sind nur im Text zitierte Titel anzuführen. Die Angaben erscheinen in alphabetischer Reihenfolge nach Verfasser. Bei mehreren Werken eines Verfassers sind diese chronologisch, beginnend mit dem ältesten, zu ordnen. Bei mehreren Publikationen eines Verfassers aus einem Jahr sind diese mit zusätzlichen Buchstaben (2009a) zu unterscheiden. Die Zitierweise orientiert sich an dem Standard der American Psychological Association (APA Style):

Artikel: Name, Vornameinitialen (Jahr). Titel. Zeitschrift, Jahrgang (Heftnummer), Seiten. Beispiel: Georg, J. (2009). Schmerzchronifizierung. NOVAcura, 40 (4), 114-117.

Mehrautorenartikel: Name, Vornameinitialen; Name, Initiale (Jahr). Titel. Zeitschrift, Jahrgang (Heftnummer), Seiten.

Beispiel: Kolbe, N.; Schnepp, W.; Zegelin, A. (2009). Leben mit chronischer Herzinsuffizienz. Pflege, 22 (2), 95-103.

Bücher: Name, Initiale(n) (Jahr). Buchtitel. Ort: Verlag.

Beispiel: Watkins, P. N. (2009). Recovery - wieder genesen können. Bern: Hans Huber.

Mehrautorenwerk: Name, Vornameinitialen; Name, Vornameinitialen (Jahr). Buchtitel. Ort: Verlag.

Beispiel: Stefanoni, S.; Alig, B. (2009). Pflegekommunikation. Bern: Hans Huber.

Buchbeiträge: Name, Vornameinitialen (Jahr). Beitrag. In: Name, Vornameinitialen, Titel. Ort: Verlag.

Beispiel: Marquard, S. (2008). Körperbild und verändertes Körperbild. In: Eicher, M.; Marquard, S. (Hrsg.), Brustkrebs. Bern: Hans Huber.

Herausgeberwerk: Name, Vornameinitialen; Name, Vornameinitialen (Hrsg.). (Jahr). Buchtitel. Ort: Verlag.

Beispiel: Panfil, E.-M.; Schröder, G. (Hrsg.) (2009). Pflege von Menschen mit chronischen Wunden. Bern: Hans Huber.

Internetquellen: Autor/Autorin, Vornameinitialen: Titel. 
http://www.vollständige_adresse/pfad/datei.html [Datum des Zugriffs].

Englischsprachige Literaturangaben: Im Englischen wird in unabhängigen Titeln (Buchtitel, Zeitschrift) bis auf Präpositionen, Artikel und Pronomen alles groß geschrieben; die abhängigen (Kapitel, Beiträge) beginnen mit einem Großbuchstaben, werden dann wie ein gewöhnlicher englischer Satz geschrieben, d. h. außer Personen- und Ortsbezeichnungen und dem Personalpronomen «I» (ich) wird alles klein geschrieben. Bei Übersetzungswerken aus dem Englischen die Original-Literaturangaben in der vorgegebenen Form konsistent übernehmen; auch nicht «ed.» durch «Hrsg.», «p.» oder «pp.» durch «S.» oder «Ss.», «and» oder «\&» durch «und» ersetzen.

\subsection{Punkt-für-Punkt-Bearbeitungsprotokoll}

Hat das Manuskript den externen Begutachtungsprozess passiert und die Redaktion bittet die Autor(inn)en, eine Überarbeitung entlang der Empfehlungen der Redaktion und/oder der Referees vorzunehmen, dann stellt das Punkt-für-Punkt-Bearbeitungsprotokoll einen wichtigen Bestandteil der Bearbeitung dar. Es bildet die Grundlage des wissenschaftlichen Diskurses über das Manuskript und ist somit ein zentrales Element im Prozess des wissenschaftlichen Publizierens. Es dient sowohl den Autor(inn)en wie den Referees zur Begründung und Orientierung in der Überarbeitung.

Grundvoraussetzung für einen konstruktiven Dialog sind Sachlichkeit und Höflichkeit. Ausgehend von der Annahme, dass die Empfehlungen der Referees prinzipiell von der Idee geleitet sind, zu einer Aufwertung, Präzisierung und inhaltlichen Schärfung des Manuskriptes zu führen, schafft ein einführender Satz wie z. B. «Wir bedanken uns für die wertvollen Anregungen der Gutachter und hoffen, diese in unserer Überarbeitung konstruktiv würdigen zu können» ein angenehmes Arbeitsklima und anerkennt den Beitrag der Referees, die unentgeltlich ihre Expertise bereitstellen.

\section{Inhalt, Vollständigkeit und Darstellung}

In dem Punkt-für-Punkt-Bearbeitungsprotokoll muss auf alle Punkte, die von der Redaktion und den Referees thematisiert wurden, chronologisch eingegangen werden. Die Autor(inn)en sollen darlegen, wie sie die Empfehlungen eingearbeitet haben. Es müssen nicht zwangsläufig alle Empfehlungen umgesetzt werden, doch dies muss inhaltlich gut begründet werden.

Für eine eindeutige Zuordnung und Orientierung ist die Nennung der Manuskriptseite beziehungsweise des Absatzes, in den die Referee-Empfehlung eingearbeitet wurde, notwendig.

\section{Einige Hilfestellungen}

$\square$ Versuchen Sie, präzise und knapp darzulegen, wie Sie auf die Empfehlung reagiert haben. $\square$ Verfassen Sie bei Nicht-Übereinstimmung keine Widerlegung oder Rechtfertigung, sondern lassen Sie das fachliche Argument sprechen.

$\square$ Versuchen Sie, die Emotionen aus dem Punkt-für-Punkt-Bearbeitungsprotokoll heraus zu halten, auch wenn der/die Referee einen scharfen oder strengen Ton angeschlagen hat. Seien Sie jedoch auch nicht zurückhaltend, wenn Sie anderer Meinung sind und klären Sie eventuelle Missverständnisse.

Behalten Sie Ihr Manuskript unter Kontrolle. Reichern Sie es nicht mit zahlreichen neuen Daten und Argumentationen an, um die Referees zufriedenzustellen. Entscheiden Sie, wann Nachbesserung und wann «nur» Argumentation notwendig ist.

$\square$ Einige Anmerkungen der Referees sind schlicht nicht umsetzbar oder führen zu weit. Hier ist Ihre klare, freundliche und gegebenenfalls literaturgestützte Argumentation vonnöten. Vermeiden Sie jedoch das Zurückhalten von Daten, wenn es Sinn machen würde, sie in diesem Manuskript zu berichten. 


\title{
Beispiele
}

\begin{tabular}{|l|l|l|}
\hline Anmerkung des Referees & Antwort der Autor(inn)en & $\begin{array}{l}\text { Änderungen im } \\
\text { Manuskript }\end{array}$ \\
\hline $\begin{array}{l}\text { Es fehlt eine Definition für } \\
\text { «freiheitseinschränkende } \\
\text { Maßnahmen». }\end{array}$ & $\begin{array}{l}\text { Vielen Dank für den Hinweis, } \\
\text { die Definition wurde ergänzt. }\end{array}$ & $\begin{array}{l}\text { Freiheitseinschränkende } \\
\text { Maßnahmen sind definiert ... } \\
\text { (S. 7). }\end{array}$ \\
\hline $\begin{array}{l}\text { Es sollte eine Tabelle mit den } \\
\text { Charakteristika der } \\
\text { Bewohner eingefügt werden. }\end{array}$ & $\begin{array}{l}\text { Aus datenschutzrechtlichen } \\
\text { Gründen konnten die } \\
\text { Charakteristika der } \\
\text { Bewohner nicht erhoben } \\
\text { werden, daher kann diese } \\
\text { Information hier nicht } \\
\text { berichtet werden. Ein } \\
\text { entsprechender Hinweis } \\
\text { wurde eingefügt. }\end{array}$ & $\begin{array}{l}\text { Aus datenschutzrechtlichen } \\
\text { Gründen konnten die } \\
\text { Bewohneristika der } \\
\text { werden (S. 5). }\end{array}$ \\
\hline $\begin{array}{l}\text { Warum wird die neben der } \\
\text { Hauptstudie durchführte } \\
\text { Erhebung zur Belastung } \\
\text { Pflegender nicht } \\
\text { ausführlicher berichtet? }\end{array}$ & $\begin{array}{l}\text { Eine Publikation zum Thema } \\
\text { befindet sich in } \\
\text { Vorbereitung, daher haben } \\
\text { wir auch angesichts der } \\
\text { Beschränkungen der } \\
\text { Manuskriptlänge auf eine } \\
\text { ausführliche Darstellung } \\
\text { dieses Nebenaspekts } \\
\text { verzichtet. }\end{array}$ & Keine Änderungen. \\
\hline
\end{tabular}

Ausführungen teilweise angelehnt an:

((Autor/Autorin)) (2010). Making your point-by-point. Editorial. Nature Structural \& Molecular Biology, 17: 389.

\section{Annahme und Veröffentlichung von Manuskripten}

Entscheidet die zuständige Herausgeberin, dass das Manuskript veröffentlicht werden soll, geht dieses zur formalen Kontrolle an das Editorische Controlling, wonach durch die Autor(inn)en die allenfalls noch notwendigen Korrekturen und Ergänzungen vorzunehmen sind. Ab diesem Zeitpunkt befindet sich der Artikel im POOL-Ordner, aus welchem die Herausgeberinnen die nächsten Hefte zusammenstellen. Sobald die Heftzuteilung erfolgt ist, werden die Autor(inn)en benachrichtigt und informiert, wann sie vom Verlag das Gutzum-Druck (GzD) zum Korrekturlesen erhalten werden. Bei endgültiger Annahme des Manuskripts ist das unterzeichnete Dokument «Autor(inn)enagreement» einzureichen (siehe Punkt 2.3).

\author{
Manuskripte für Dissertationen umfassen \\ Titel der Dissertation \\ Autor(inn)en und Angliederung (Institution) \\ Einleitung (mit Ziel und Zweck) \\ Methoden \\ Ergebnisse \\ Schlussfolgerungen (Relevanz für die \\ Pflegepraxis) \\ Umfang: etwa 5000 Zeichen inklusive Leerschläge
}

Die Formatierung erfolgt in der gleichen Weise wie für Manuskripte.

\section{Buchbesprechungen}

Der Umfang von Buchbesprechungen beträgt 2 Spalten oder 3000 Zeichen (inklusive Leerzeichen). Die Formatierung erfolgt in der gleichen Weise wie für Manuskripte. Die bibliografischen Angaben umfassen: Autor(in), Titel, Jahr, Ort, Verlag, Seiten (Abbildungen, Tabellen), Preis und ISBN. Buchbesprechungen können als Word-Datei per Mail unter folgender Adresse eingereicht werden: rosemarie.voelkle@usz.ch. 
Der Verlag ist auch an Buchpublikationen zum Thema Pflege interessiert. Wenn Sie Vorschläge oder eigene Publikationsprojekte haben, wenden Sie sich an:

Jürgen Georg Verlag Hans

Huber, Hogrefe AG Lektorat

Pflege Länggass-Straße $76 \mathrm{CH}$ -

3000 Bern 9

juergen.georg@hanshuber.com 


\subsection{Agreement Autor(inn)en}

Ich bestätige folgende Aussagen: [ ] Alle als Autor(inn)en genannten Personen sind mit den Inhalten und der Veröffentlichung einverstanden. [ ] Die genannten Beiträge der einzelnen Autor(inn)en zur Arbeit werden als korrekt bestätigt. [ ] Der Beitrag ist ein Originalartikel und wurde noch nicht in einem anderen Journal, einem anderen Buch oder im Internet veröffentlicht. [ ] Der Beitrag ist nicht zeitgleich in gleicher oder vergleichbarer Form oder in Auszügen bei einer anderen Zeitschrift oder als Beitrag zu einem Buch oder einer Website zur Publikation eingereicht worden oder bereits erschienen. [ ] Die Autor(inn)en bestätigen, dass sie uneingeschränkt über sämtliche Urheberrechte an ihrem Beitrag einschließlich eventueller Bildvorlagen, Zeichnungen, Pläne, Karten, Skizzen und Tabellen verfügen und dass der Beitrag keine Rechte Dritter verletzt. Befinden sich bereits andernorts publizierte Auszüge einer (auch eigenen) Veröffentlichung (z.B. Tabelle, Abbildung) im Manuskript, wurde die Reproduktionsgenehmigung des entsprechenden Verlages von den Autor(inn)en eingeholt und der Redaktion vorgelegt. [ ] Die Autor(inn)en sind darüber informiert, dass im Falle eines Plagiats weitere Publikationen aller am Manuskript beteiligten Autor(inn)en für die «Pflege» gesperrt werden können.

[ ] Mit der Annahme eines Manuskripts geht das Verlagsrecht entsprechend den gesetzlichen Bestimmungen an den Verlag Hans Huber, Bern, über. [ ] Die Autor(inn)en räumen - und zwar auch zur Verwertung ihres Beitrags außerhalb der inn enthaltenen Zeitschrift und unabhängig von deren Veröffentlichung - dem Verlag räumlich und mengenmäßig unbeschränkt für die Dauer des gesetzlichen Urheberrechts das ausschließliche Recht der Vervielfältigung und Verbreitung bzw. der unkörperlichen Wiedergabe des Beitrages ein. Die Autor(inn)en räumen dem Verlag ferner die folgenden ausschließlichen Nutzungsrechte am Beitrag ein:

a) das Recht zum ganzen oder teilweisen Vorabdruck und Nachdruck - auch in Form eines Sonderdrucks, zur Übersetzung in andere Sprachen, zu sonstiger Bearbeitung und zur Erstellung von Zusammenfassungen (Abstract);

b) das Recht zur Veröffentlichung einer Mikrokopie-, Mikrofiche- und Mikroformausgabe, zur Nutzung im Weg von Bildschirmtext, Videotext und ähnlichen Verfahren, zur Aufzeichnung auf Bild- und/oder Tonträger und zu deren öffentlicher Wiedergabe - auch multimedial - sowie zur öffentlichen Wiedergabe durch Radio- und Fernsehsendungen;

c) das Recht zur maschinenlesbaren Erfassung und elektronischen Speicherung auf einem Datenträger (z. B. Diskette, CD-ROM, Magnetband) und in einer eigenen oder fremden Online-Datenbank, zum Download in einem eigenen oder fremden Rechner, zur Wiedergabe am Bildschirm - sei es unmittelbar oder im Weg der Datenfernübertragung , sowie zur Bereithaltung in einer eigenen oder fremden Online-Datenbank zur Nutzung durch Dritte;

d) das Recht zu sonstiger Vervielfältigung, insbesondere durch fotomechanische und ähnliche Verfahren (z. B. Fotokopie, Fernkopie) und zur Nutzung im Rahmen eines sogenannten Kopienversands auf Bestellung;

e) das Recht zur Vergabe der vorgenannten Nutzungsrechte an Dritte im In- und Ausland sowie die von der Verwertungsgesellschaft WORT und Pro Litteris wahrgenommenen Rechte einschließlich der entsprechenden Vergütungsansprüche.

Name des Erstautors/der Erstautorin in Blockschrift:

Titel des Beitrags:

Datum, Ort: Unterschrift:

Per Post einsenden an: Frau Rosemarie S. Völkle, UniversitätsSpital Zürich, Rämistr. 100 (ZUR 44), CH-8091 Zürich, oder als Datei per Mail an rosemarie.voelkle@usz.ch oder per Fax an +41(0)442554395 